Case Report

\title{
Treatment of Nonclassic 11-Hydroxylase Deficiency with Ashwagandha Root
}

\author{
Daniel Powell, Taiga Inoue, Gül Bahtiyar, Gabriel Fenteany, and Alan Sacerdote \\ Department of Medicine, New York City Health + Hospitals/Woodhull, 760 Broadway, Brooklyn, NY 11206, USA \\ Correspondence should be addressed to Alan Sacerdote; alan.sacerdote@woodhullhc.nychhc.org
}

Received 25 February 2017; Accepted 21 May 2017; Published 20 June 2017

Academic Editor: Michael P. Kane

Copyright (c) 2017 Daniel Powell et al. This is an open access article distributed under the Creative Commons Attribution License, which permits unrestricted use, distribution, and reproduction in any medium, provided the original work is properly cited.

\begin{abstract}
An elderly woman presented with acne and male pattern alopecia, which upon diagnostic evaluation was found to be due to nonclassic 11-hydroxylase deficiency. We previously reported that Ashwagandha root ameliorates nonclassic 3- $\beta$-ol dehydrogenase and aldosterone synthase deficiencies. This is the first report of its use being associated with amelioration of nonclassic 11hydroxylase deficiency, where its apparent effects appear to be dose-related.
\end{abstract}

\section{Introduction}

Congenital adrenal hyperplasia $(\mathrm{CAH})$, like PCOS, is characterized by insulin resistance [1-5]. The Ayurvedic herb, Ashwagandha, (Withania somnifera), also known as Indian ginseng, has been used for millennia with numerous beneficial health effects having been reported; it helps fight infections, deters oil plugs from being formed on the skin, and is a natural and healthy way to treat acne scars [6, 7]. A newer usage of Ashwagandha root is as an insulin sensitizer [8]. Hyperinsulinemia may exert its redirection of adrenal steroidogenesis to androgens and away from cortisol, via its effects on the synthesis and expression of steroidogenic factor-1 (SF-1) and the nuclear transcription factor Nur77 [9]. Our group has reported a number of interventions known to decrease insulin resistance or hyperinsulinemia, which ameliorate both classic and nonclassic $\mathrm{CAH}$ and include metformin, thiazolidinediones, weight loss by way of lifestyle change or bariatric surgery, vitamin D replacement, and supplementation with Ashwagandha (Withania somnifera) [10-16]. Conversely, we have also reported that drugs known to cause insulin resistance and, in some instances, PCOS have also been shown to induce the expression of nonclassic adrenal hyperplasia [17]. Such drugs include valproate, classical and atypical antipsychotics, and antiretroviral drugs. In our previous experience with Ashwagandha, we reported that it normalized elevated serum levels of corticosterone and 17-hydroxypregnenolone and ameliorated acne and excessive scalp hair loss in a patient with both nonclassic aldosterone synthase deficiency and 3- $\beta$-ol dehydrogenase deficiency [14]. Here, we share our experience in treating a patient with nonclassic 11-hydroxylase deficiency with Ashwagandha.

\section{Case Presentation}

A 78-year-old G1P1001 woman with a history of hypothyroidism, hypertension, and stage III chronic kidney disease presented with a complaint of long-standing acne and alopecia. Her growth, developmental, and reproductive history were unremarkable; her complaints began in her seventies. On 6/18/13, her unstimulated serum 11-deoxycortisol concentration was impressively elevated at $91 \mathrm{ng} / \mathrm{dL}(<37 \mathrm{ng} / \mathrm{dL})$ consistent with a nonclassic 11-hydroxylase deficiency. Serum 11-deoxycortisol concentration was determined by liquid chromatography/tandem mass spectrometry. Cosyntropin (ACTH) stimulation testing was considered superfluous in this setting, as the baseline serum 11-deoxycortisol concentration was already $2.5 \times$ the upper limit of the reference range and other adrenal steroid metabolites (17$\mathrm{OH}$-progesterone, 17-OH-pregnenolone, and deoxycorticosterone) were all within normal limits and because (unlike 17-OH-progesterone) there is no quantitatively important ovarian source for 11-deoxycortisol. The patient was not treated with metformin, which is usually effective in $\mathrm{NCAH}$ $[10,15]$, because of her stage $3 \mathrm{~b}$ chronic kidney disease. Based on our experience with other forms of nonclassic congenital 


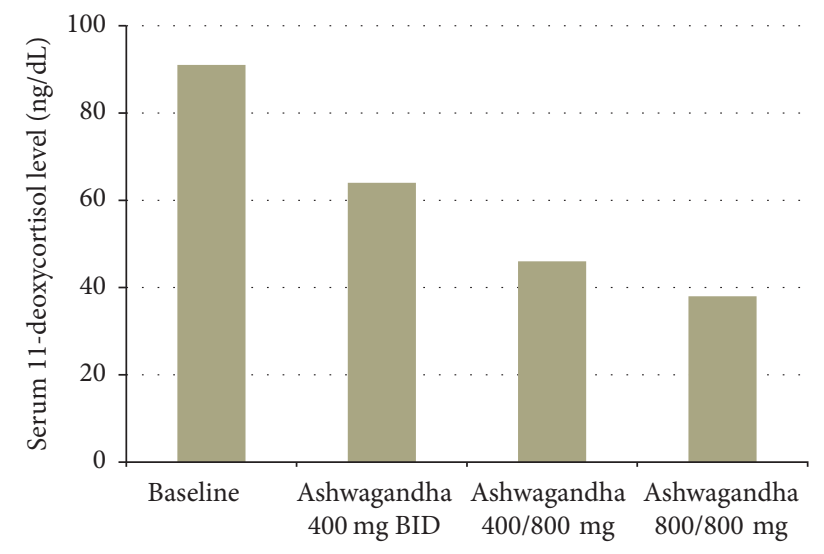

FIGURE 1: Effect of Ashwagandha root on serum 11-deoxycortisol (ng/dl).

adrenal hyperplasia and honoring the patient's request to try a "natural" approach, after obtaining informed consent, treatment was initiated with a standardized preparation of Ashwagandha root, with a dosage of $400 \mathrm{mg}$ twice daily, which is the starting dose recommended on the product label as well as the dose that we previously reported as effective in treating other forms of NCAH [14]. On 4/9/2014, her serum 11-deoxycortisol concentration declined to $64 \mathrm{ng} / \mathrm{dL}$. The dosage was increased to $400 \mathrm{mg}$ to be taken in the morning and $800 \mathrm{mg}$ in the evening. On 12/12/2014, the serum 11-deoxycortisol level further declined to $46 \mathrm{ng} / \mathrm{dL}$. We then increased the dosage to $800 \mathrm{mg}$ twice daily. On $5 / 3 / 16$, the serum 11-deoxycortisol was $33 \mathrm{ng} / \mathrm{dL}$. We noted an apparent dose/effect relationship between the Ashwagandha dosage and serum 11-deoxycortisol concentration. After a period of three years on a gradually increasing dose of Ashwagandha root, her serum 11-deoxycortisol concentration had normalized (Figure 1). Biochemical improvement has been accompanied by resolution of acne and reduction in hair loss. Since her acne was primarily facial, we chose not to photograph her, even with her eyes covered, to protect her privacy. For safety monitoring, we followed up the patient's vital signs, serum glucose, electrolytes, complete blood count, liver function tests, lipid profile, urinalysis, estimated glomerular filtration rate, and microalbumin, none of which changed appreciably.

\section{Discussion}

Ashwagandha root, often referred to as Indian ginseng, was first recorded for medical use by the Ayurveda $[6,7]$. Today, it is widely grown in tropical and subtropical areas. It has been prescribed for a multiplicity of indications over several millennia and is considered by some as an adaptogen (an herb, molecule, or substance that promotes homeostasis). The first report of Ashwagandha root acting as an insulin sensitizer was by Anwer et al. [8]. As mentioned previously, we have reported amelioration of $\mathrm{CAH}$ through treatment with a number of insulin-sensitizing interventions including metformin, lifestyle changes, bariatric surgery, thiazolidinediones, and vitamin D repletion $[10-13,15]$. Previously, we reported amelioration of two other forms of NCAH with Ashwagandha root [14]. The present report adds additional evidence to the already formidable volume of evidence that, as in PCOS, insulin resistance is not merely a bystander in $\mathrm{CAH}$, but rather an integral part of its pathophysiology, thus informing an approach to its treatment. As discussed above, cosyntropin stimulation testing was unnecessary in this instance for the reasons cited in the Case Presentation; such testing is necessary, however, when there is a strong clinical suspicion of NCAH in the face of normal baseline levels of key adrenal metabolites. In such situations, cosyntropin testing may unmask those with functionally milder homozygous mutations and many heterozygotes

Withanolides are a class of compounds extracted from Ashwagandha root, and they have the ability to modulate the activity of GABAergic receptors [16]. GABAergic receptors are widely distributed in the body, including in the adrenal cortex [18]; they exert an effect on adrenal steroidogenesis [19], which could ameliorate the expression of inherited or acquired disorders of steroidogenesis. Guidelines for adult dosing of Ashwagandha have been reported [20].

Although acne in the elderly is somewhat uncommon, it is by no means unheard of [21], and when it is encountered, it is usually long-standing. Although our patient is quite elderly, there needs to be no concern about her ability to provide informed consent. She lives independently in her own home with her partner, functions independently in all activities of daily living, has no apparent cognitive impairment, and makes the 1.5-hour trip to our clinic alone by commuter train and subway. She also routinely uses the web to search for health-related information and is active on social media.

In summary, we report that Ashwagandha root may be an effective treatment for some patients with nonclassic 11hydroxylase deficiency. Since Ashwagandha has been shown to be an insulin sensitizer, the apparent response seen here lends further support to the growing body of evidence that insulin resistance, far from being a mere side product of CAHs, is actually an integral part of their pathophysiology and a potential pathway to their treatment. As has been shown with lifestyle changes, bariatric surgery, metformin, vitamin $\mathrm{D}$ repletion, and thiazolidinediones, Ashwagandha use is associated with amelioration of at least some forms of $\mathrm{CAH}$, without the adverse effects of glucocorticoids. The ability of withanolides to affect the activity of GABAergic receptors and the presence of the latter in the adrenal cortex raises the possibility that these compounds could ameliorate inherited and acquired defects of adrenal steroidogenesis.

\section{Disclosure}

An earlier version of this work was presented as a poster at the Endocrine Society's 97th Annual Meeting and Expo, March 5-8, San Diego, CA, USA, which may be accessed at http:// press.endocrine.org/doi/abs/10.1210/endo-meetings.2015.AH -PAA.7THR-416. Three authors of the above abstract, Drs. Giunta, George, and Baby, did not participate further in the preparation of this manuscript after the above poster presentation. 


\section{Conflicts of Interest}

The authors declare that there are no conflicts of interest regarding the publication of this paper.

\section{Authors' Contributions}

Alan Sacerdote, Gül Bahtiyar, and Taiga Inoue participated in the care of the patient, data collection, literature review preparation of the figure, and editing of the manuscript. Daniel Powell performed literature review, wrote the first draft of the manuscript, and did some additional editing of the manuscript. Gabriel Fenteany did additional editing of the manuscript and figure and participated in literature review and actually submitted the manuscript.

\section{References}

[1] P. W. Speiser, J. Serrat, M. I. New, and J. M. Gertner, "Insulin insensitivity in adrenal hyperplasia due to nonclassical steroid 21-hydroxylase deficiency," Journal of Clinical Endocrinology and Metabolism, vol. 75, no. 6, pp. 1421-1424, 1992.

[2] F. Saygili, A. Oge, and C. Yilmaz, "Hyperinsulinemia and insulin insensitivity in women with nonclassical congenital adrenal hyperplasia due to 21-hydroxylase deficiency: the relationship between serum leptin levels and chronic hyperinsulinemia," Hormone Research, vol. 63, no. 6, pp. 270-274, 2005.

[3] E. Charmandari, M. Weise, S. R. Bornstein et al., "Children with classic congenital adrenal hyperplasia have elevated serum leptin concentrations and insulin resistance: potential clinical implications," Journal of Clinical Endocrinology and Metabolism, vol. 87, no. 5, pp. 2114-2120, 2002.

[4] M. F. Mnif, M. Kamoun, F. Mnif, and etal., "Long-term outcome of patients with congenital adrenal hyperplasia due to 21hydroxylase deficiency," The American Journal of the Medical Sciences, vol. 344, no. 5, pp. 363-373, 2012.

[5] W. Arlt, D. S. Willis, S. H. Wild, N. Krone et al., "Health status of adults with congenital adrenal hyperplasia: a cohort study of 203 patients," The Journal of Clinical Endocrinology \& Metabolism, vol. 95, no. 11, pp. 5110-5121, 2010.

[6] F. Narenda, M. Balla, J. Prashinta, and M. Gilca, "An overview on ashwagandha: a rasayana (rejuvenator) of ayurveda," African Journal of Traditional, Complementary and Alternative Medicines, vol. 8, supplement 5, pp. 208-2013, 2011.

[7] http://www.chiro.org/nutrition/Ashwagandha.shtml\#Research.

[8] T. Anwer, M. Sharma, K. K. Pillai, and M. Iqbal, "Effect of Withania somnifera on insulin sensitivity in non-insulin-dependent diabetes mellitus rats," Basic and Clinical Pharmacology and Toxicology, vol. 102, no. 6, pp. 498-503, 2008.

[9] S. N. Kelly, T. J. McKenna, and L. S. Young, "Modulation of steroidogenic enzymes by orphan nuclear transcriptional regulation may control diverse production of cortisol and androgens in the human adrenal," The Journal of Endocrinology, vol. 181, no. 2, pp. 355-365, 2004.

[10] G. Bahtiyar and A. Chatterjee, "Management approaches to congenital adrenal hyperplasia in adolescents and adults; latest therapeutic developments," in Amenorrhea, A. Chatterjee, Ed., p. 148, InTech, December 2011.

[11] A. Kalani, N. Thomas, A. Sacerdote, and G. Bahtiyar, "Rouxen-Y gastric bypass in the treatment of non-classic congenital adrenal hyperplasia due to 11-hydroxylase deficiency," BMJ Case Reports, vol. 2013, 2013.

[12] N. Thomas, A. Kalani, R. Vincent et al., "Effect of vitamin D in a patient with classical adrenal hyperplasia due to 11-hydroxylase deficiency," Journal of Medical Cases, vol. 4, no. 8, pp. 569-575, 2013.

[13] M. Vitiello, G. Bahtiyar, and A. Sacerdote, "Vitamin D deficiency/insufficiency in patients with non-classic adrenal hyperplasia and response of the latter to vitamin D replacement," Endocrine Reviews, vol. 33, no. 3, 2012.

[14] A. Kalani, G. Bahtiyar, and A. Sacerdote, "Ashwagandha root in the treatment of non-classical adrenal hyperplasia," BMJ Case Reports, vol. 2012, 2012.

[15] A. Sacerdote and G. Bahtiyar, "Treatment of congenital adrenal hyperplasia by reducing insulin resistance and cysticercosis induced polycystic ovarian syndrome," in Contemporary Gynecologic Practice, A. Darwish, Ed., chapter 5, February 2015.

[16] A. C. Mapas-Dimaya, L. Agdere, G. Bahtiyar, J. O. Mejia, and A. S. Sacerdote, "Metformin-responsive classic salt-losing congenital adrenal hyperplasia due to 21-hydroxylase deficiency: a case report," Endocrine Practice, vol. 14, no. 7, pp. 889-891, 2008.

[17] G. Bahtiyar, K. Weiss, and A. S. Sacerdote, "Novel endocrine disrupter effects of classic and atypical antipsychotic agents and divalproex: induction of adrenal hyperandrogenism, reversible with metformin or rosiglitazone," Endocrine Practice, vol. 13, no. 6, pp. 601-608, 2007.

[18] M. Candelario, E. Cuellar, J. M. Reyes-Ruiz et al., "Direct evidence for GABAergic activity of Withania somnifera on mammalian ionotropic GABAA and GABA $\rho$ receptors," Journal of Ethnopharmacology, vol. 171, pp. 264-272, 2015.

[19] K. Metzeler, A. Agoston, and M. Gratzl, "An intrinsic $\gamma$-aminobutyric acid (GABA)ergic system in the adrenal cortex: findings from human and rat adrenal glands and the NCI-H295R cell line," Endocrinology, vol. 145, no. 5, pp. 2402-2411, 2004.

[20] https://www.drugs.com/npp/ashwagandha.html.

[21] D. C. Seukeran and W. J. Cunliffe, "Ache vulgaris in the elderly: the response to low-dose isotretinoin," British Journal of Dermatology, vol. 139, no. 1, pp. 99-101, 1998. 


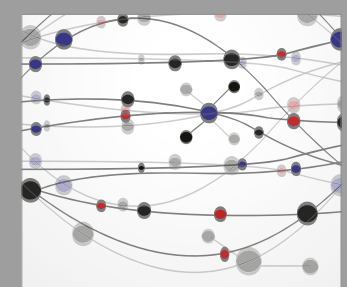

The Scientific World Journal
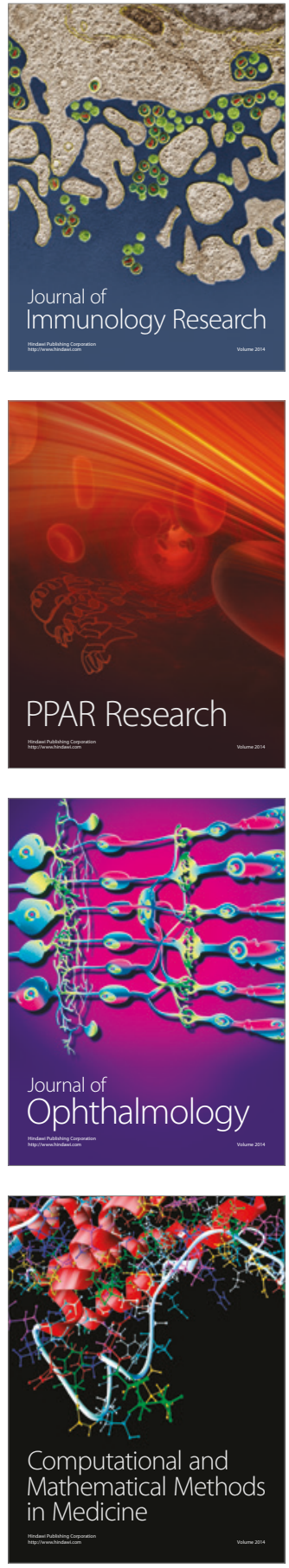

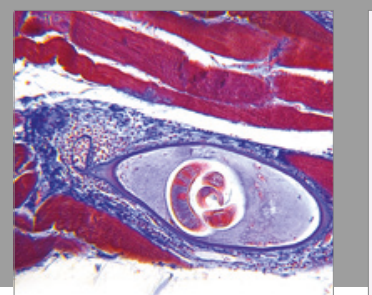

Gastroenterology Research and Practice
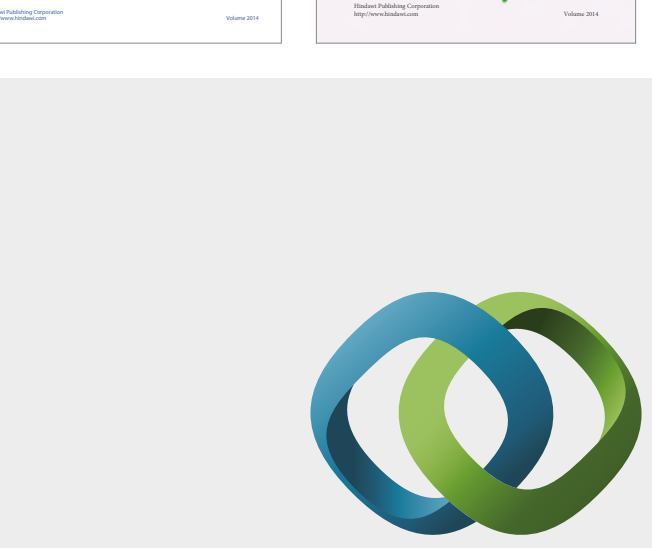

\section{Hindawi}

Submit your manuscripts at

https://www.hindawi.com
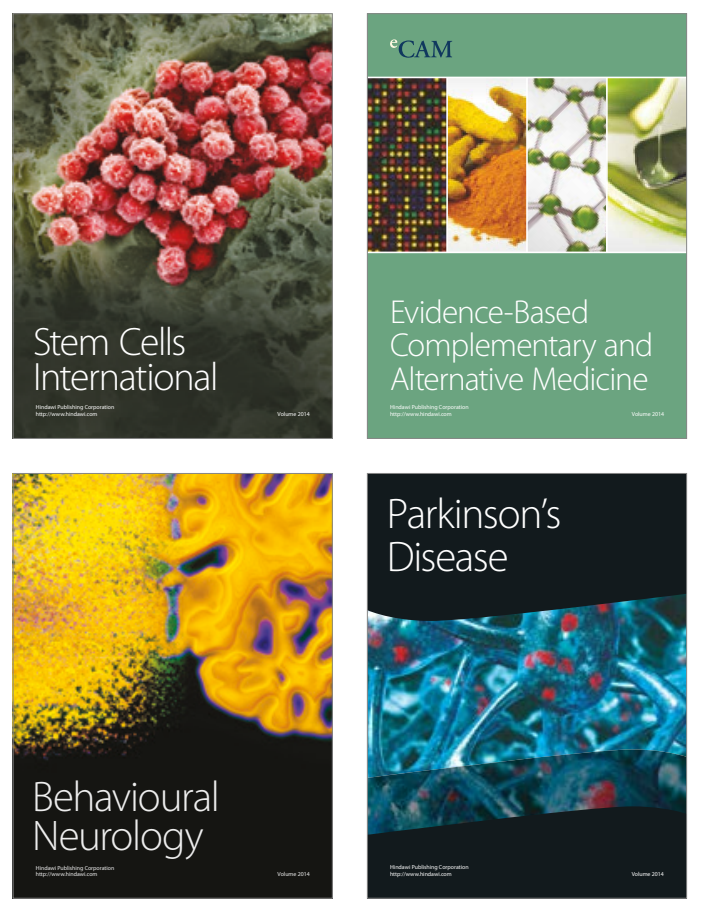
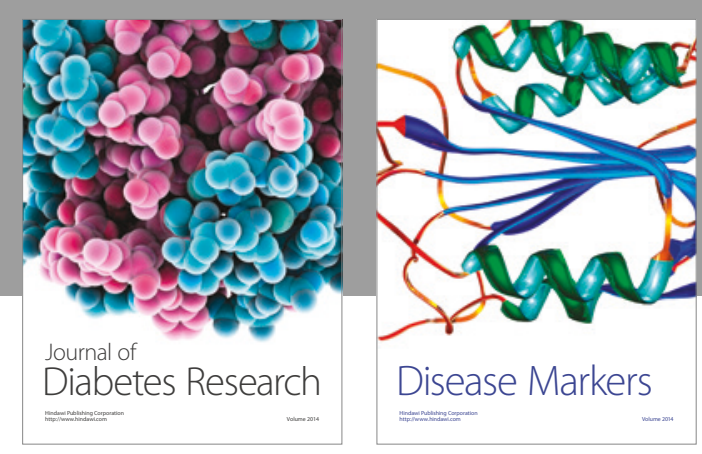

Disease Markers
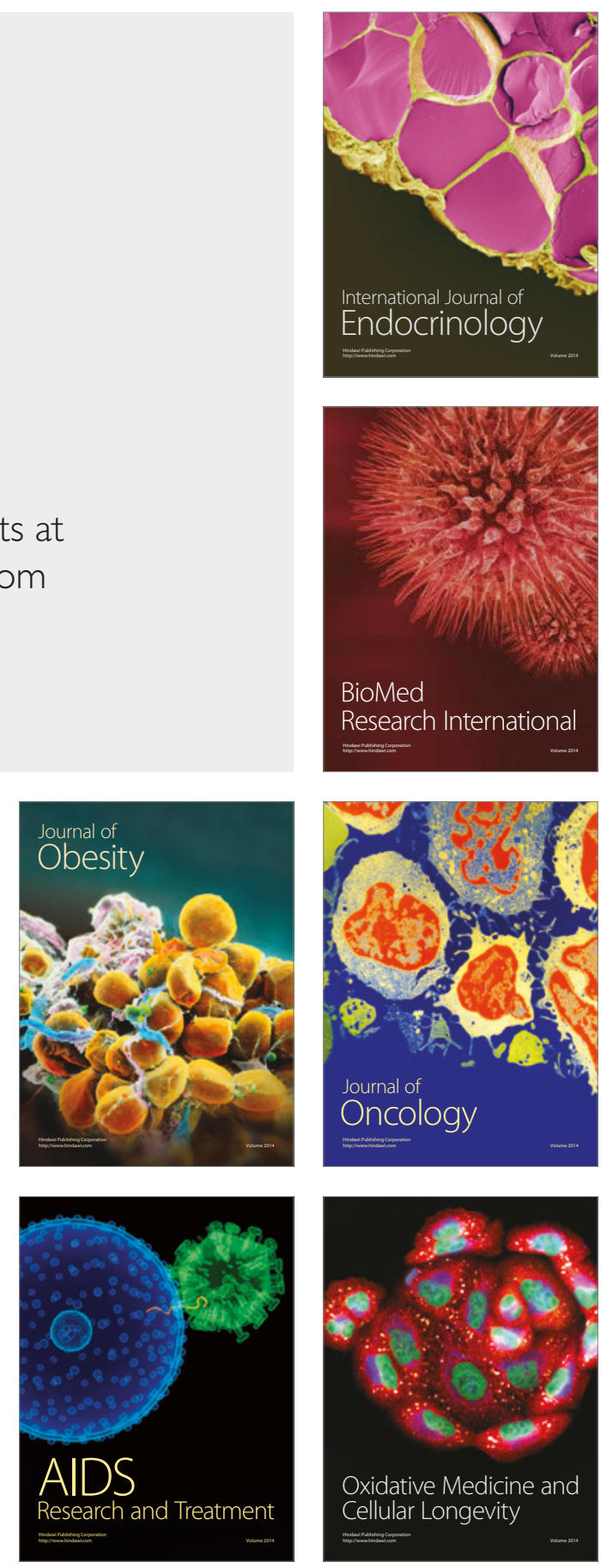